\title{
La entonación declarativa e interrogativa en el español colombiano de Medellín: voz femenina vs. masculina
}

\author{
Mercedes Muñetón Ayala \\ Universidad de Antioquia, Colombia \\ Josefa Dorta Luis \\ Universidad de La Laguna, España
}

\begin{abstract}
Resumen
Este trabajo se ha realizado en el marco del Proyecto AMPER-Col (Atlas Multimedia de Prosodia del Espacio Románico en Colombia). El objetivo del presente trabajo es analizar los contornos entonativos, declarativos e interrogativos, de la voz femenina y masculina de dos hablantes colombianos pertenecientes a la zona urbana de Medellín. Los resultados muestran que hay rasgos diferenciadores entre ambos tipos de voz, como la ubicación de los picos máximos, la pendiente Inicio-PMx1, PMx-Final y la pendiente Inicio-Final, lo que tiene implicaciones fonéticas y fonológicas. No obstante, los datos deben tomarse con cautela, dado que este es uno de los primeros acercamientos al análisis de la prosodia en Colombia.

Palabras clave: prosodia, entonación, frecuencia fundamental, fonética y fonología de la entonación.
\end{abstract}

\footnotetext{
Para correspondencia, dirigirse a: Dra. Mercedes Amparo Muñetón Ayala <mamuneton@ hotmail.com>, Universidad de Antioquia, Facultad de Comunicaciones, Departamento de Filología Hispánica, Calle 67 Número 53 - 108, Medellín, Antioquia, Colombia, o a Dra. Josefa Dorta<jdorta@ull.edu.es>, Universidad de La Laguna, Facultad de Humanidades (sección Filología), Departamento de Filología Española, Laboratorio de Fonética, Campus de Guajara s/n. CP76081, La Laguna, S/C de Tenerife, Islas Canarias/España.
} 


\title{
INTONATION OF DECLARATIVE AND INTERROGATIVE STATEMENTS IN \\ Colombian Spanish of Medellin: female VS. Male voices
}

\begin{abstract}
This research has been carried out within the framework of AMPER-Col (Multimedia Atlas of Prosody of the Romanic Space in Colombia). The main objective is to analyze the intonation contours of declaratives and interrogatives of female and male voices of two Colombian speakers belonging to the urban zone of Medellin. Results show us that there are differences between these voices in the maximum peak location, initial-PMx1 slope and the initial-final slope. These features have phonetic and phonological implications. However, those data should be taken with caution given that this is one of the first prosody studies in Colombia.
\end{abstract}

Keywords: prosody, intonation, fundamental frequency, phonetics and phonology of intonation.

Recibido: 20/10/14 Aceptado: $31 / 07 / 15$

\section{INTRODUCCIÓN**}

El estudio de la prosodia ha sido menos abordado que otros temas lingüísticos. Desde hace ya algunos años, este vacío está siendo subsanado, en buena medida, por el proyecto AMPER (Atlas Multimedia de Prosodia del Espacio Románico), liderado internacionalmente por Antonio Romano y Michel Contini (2001). El objetivo de este macroproyecto es analizar los aspectos de frecuencia fundamental (F0), duración e intensidad de las distintas variedades diatópicas de las lenguas románicas; en principio, el interés se centra en frases enunciativas e interrogativas de hablantes femeninos y masculinos procedentes de zonas urbanas y rurales,

** Este trabajo se enmarca en dos proyectos de investigación vinculados al proyecto internacional AMPER (Atlas Multimedia de Prosodia del Espacio Románico). El primero es "Atlas Multimedia de Prosodia del Espacio Románico en Colombia (AMPERCol): Medellín y Bogotá", concedido por el Comité para el Desarrollo de la Investigación-CODI- 2012 para el Área de Ciencias Sociales, Humanidades y Artes de la Universidad de Antioquia y dirigido por Mercedes Amparo Muñetón. El segundo es La entonación interrogativa y declarativa del español de Canarias y su relación con la de Cuba y Venezuela (Ref. PI FFI2010-16993) subvencionado por el Ministerio de Economía y Competitividad y dirigido por Josefa Dorta del Laboratorio de Fonética de la Universidad de La Laguna. 
utilizando un enfoque teórico y metodológico uniforme con el fin de crear un corpus de datos que permita hacer comparaciones y extraer conclusiones sobre una misma base.

Actualmente, en Colombia contamos con pocos trabajos de investigación que aborden el análisis de la prosodia con una metodología que arroje resultados acerca de los rasgos entonativos y acentuales del habla colombiana, y hasta el momento no hay un estudio que enmarque la prosodia de las diferentes variedades nacionales, propósito general de AMPER-Col. Uno de los pocos trabajos existentes es el realizado por Osorio y Muñoz (2011), quienes hallan los patrones entonativos a partir de muestras televisivas de las que entresacan enunciados interrogativos elípticos y no elípticos de los hablantes de español de la ciudad de Medellín. Los resultados sugieren que la entonación en los primeros es descendente, contrariamente a las interrogativas no elípticas que presentan una inflexión final ascendente. Asimismo, Ham (2003) realizó un estudio cuyo objetivo era lograr una aproximación a la comprensión del funcionamiento de curvas entonativas como índices de identidad para variantes dialectales entre informantes bogotanas y paisas. En este trabajo participaron cuatro informantes femeninas (dos paisas y dos bogotanas). Se les dio un guión y se les pidió que lo actuaran como si se tratara de una radionovela de su región. A las producciones seleccionadas se les extrajeron las melodías que luego fueron evaluadas por otros informantes como pertenecientes o no a su dialecto. Los resultados muestran que los hechos de entonación funcionan como índices de identidad dialectal. Además de los trabajos citados, la prosodia colombiana ha sido mencionada en el trabajo de Sosa (1999: 197), quien analiza los contornos entonativos de hablantes cultos en oraciones declarativas del español peninsular (Madrid, Pamplona, Barcelona y Sevilla) e hispanoamericano (Buenos Aires, Bogotá, Ciudad de México, San Juan de Puerto Rico, Caracas, La Habana y Lima). El autor sugiere que, a pesar de que existen similitudes entre dichas variedades, puesto que el tonema generalmente fue descendente, su origen se puede identificar, por lo cual concluye que "hay diferencias en la selección de acentos tonales en el pretonema".

En definitiva, los rasgos prosódicos que identifican el habla colombiana son muy escasos, por lo que se requiere un trabajo sistemático y con respaldo científico. Desde esta perspectiva, el objetivo de este estudio es comparar la entonación de la voz femenina y masculina de dos hablantes colombianos pertenecientes a la zona urbana de Medellín. Para ello, tendremos en cuenta la tipología acentual y las fronteras sintagmáticas en función de la modalidad oracional. Con los datos obtenidos haremos una interpretación fonético-fonológica de los acentos tonales. Asimismo, observaremos el nivel inicial y final del contorno entonativo respecto del tono medio, así como la pendiente inicio-final. 


\section{METODOLOGÍA}

\subsection{INFORMANTES}

Dado que los estudios de entonación colombiana en el marco de AMPER se están iniciando, este trabajo preliminar analiza un corpus de oraciones emitidas por una mujer y un hombre sin estudios superiores de zonas urbanas de Medellín, con edades comprendidas entre los 25 y 45 años. Se trata de un corpus experimental, obtenido por elicitación textual, de 108 oraciones sin expansión (54 declarativas y otras tantas interrogativas), del tipo SVO (sujeto+verbo+objeto). Los sintagmas inicial y final están compuestos por trisílabos de diferente tipología acentual (oxítona, paroxítona, proparoxítona); el sintagma central permanece invariable (acento paroxítono). Se trata de frases del tipo El saxofón se toca con paciencia; La guitarra se toca con pánico; La cítara se toca con obsesión y todas las combinaciones acentuales posibles en las fronteras inicial y final.

\subsection{ANÁlisis ACÚSTICO}

Las grabaciones se hicieron en una cabina insonorizada del laboratorio de fonética de la Universidad de Antioquia. Utilizamos el programa Goldwave 4.25 para el tratamiento de los ficheros de sonido (.wav) y estos fueron analizados posteriormente con el programa AMPER-2006 (López et al. 2007) en el entorno Matlab Matriz Laboratory ${ }^{1}$. Las rutinas creadas en Matlab permiten obtener, a partir de la segmentación en el oscilograma, tres valores de F0 de cada vocal silábica (en el inicio, en el medio y en el final).

Para delimitar las diferencias mínimas perceptibles (Just Noticeable Differences) en la F0 hay múltiples propuestas que sugieren distintos umbrales (Gelfand 1981; Klatt 1973; Pollack 1952, Rietveld y Gussenhoven, 1985, entre otros); estos últimos autores proponen para el holandés el de 1,5 semitonos (St) que posteriormente fue aplicado para el español por Toledo (2000) y por Pamies Bertrán et al. (2002). En este último estudio, un grupo de participantes, sin conocimientos previos del área de lingüística, realizaron una tarea de igual-diferente usando el estímulo /ba/; para ello escuchaban 
secuencias de la sílaba repetida dos veces (ba-ba), con la particularidad de que ambas sílabas eran iguales, excepto en la frecuencia debido a que había sido modificada de forma gradual en $1 \mathrm{St}$. Las conclusiones a las que llegan estos autores, igual que Rietveld y Gussenhoven para el holandés, es que la unidad mínima potencialmente perceptible es $1,5 \mathrm{St}$, puesto que está a medio camino entre el mínimo perceptible -1 St - y el máximo imperceptible -2 St-. La fórmula con el fin de observar las diferencias entre dos frecuencias en $\mathrm{St}$ es $\mathrm{D}=((12 / \mathrm{LOG}(2)) *(\operatorname{LOG}(\mathrm{B} 2 / \mathrm{B} 3)))$ (Nooteboom 1997: 645).

\section{RESULTADOS}

\subsection{CONFIGURACIÓN TONAL, FRONTERAS SINTAGMÁTICAS Y ACENTOS TONALES DE LAS DECLARATIVAS E INTERROGATIVAS}

Como cabía esperar, las configuraciones tonales del hombre se sitúan en un nivel tonal inferior respecto a las de la mujer (Figuras 1 y 2), independientemente de la modalidad oracional y de la tipología acentual. Las diferencias entre ambos sexos oscilan entre 9 y $10 \mathrm{St}$, desde el inicio hasta el final de la curva melódica.

\subsubsection{Modalidad declarativa}

En esta modalidad (Figura 1) el contorno entonativo de ambos informantes, independientemente de la tipología acentual, se caracteriza por presentar dos picos tonales máximos (PMx) escalonados en pendiente descendente, por lo que el contorno es bicumbre. El inicio medio (I) se sitúa en 213 y $116 \mathrm{~Hz}$, en mujer y hombre, respectivamente $\mathrm{y}$, en torno al mismo, sin que se den diferencias por encima del umbral considerado (1,5 St), la F0 comienza a ascender a partir de la pretónica (primera, segunda o tercera sílaba, dependiendo de si se trata de proparoxítonas, paroxítonas u oxítonas, respectivamente), hasta sobrepasar el tono medio $(\mathrm{TM})^{2}$ de cada informante; el ascenso culmina en el primer pico máximo (PMx1) que se pospone siempre recayendo en la postónica (Tabla 1). A continuación desciende, tanto en el hombre como en la mujer, hasta la sílaba tónica del sintagma 
verbal (SV), para luego volver a ascender hasta el segundo pico máximo $(\mathrm{PMx} 2)$ que se alinea con una átona al sincronizarse, ya sea con el final del sintagma verbal, o con el inicio del sintagma preposicional. A partir de aquí hay un descenso, más progresivo en el hombre que en la mujer, a lo largo del sintagma preposicional ubicándose el final medio de la mujer (F) en 217 $\mathrm{Hz}$ y el del hombre en $138 \mathrm{~Hz}$.

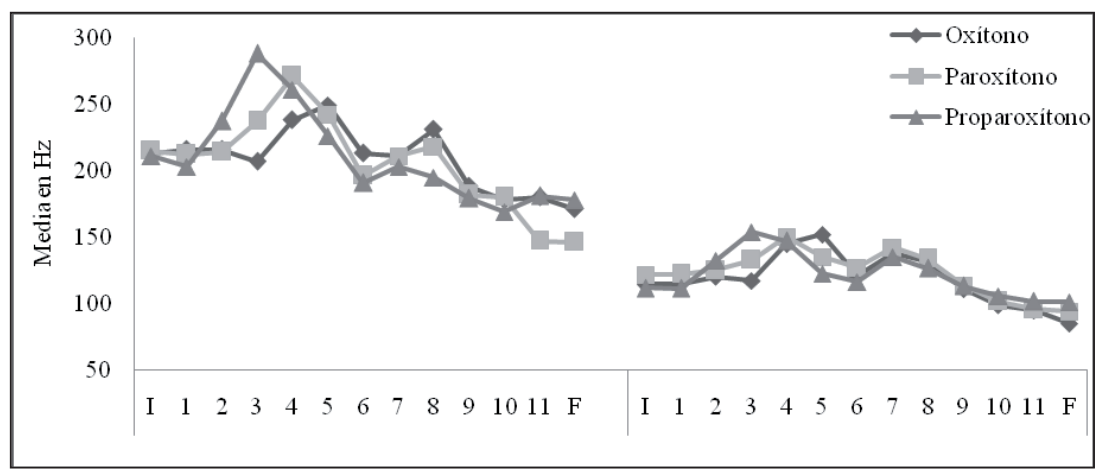

Figura 1. Modalidad declarativa en función de la tipología acentual

\begin{tabular}{|c|c|c|c|c|c|c|c|c|c|}
\cline { 3 - 10 } \multicolumn{2}{c|}{} & \multicolumn{2}{|c|}{ Valor en HZ } & \multicolumn{2}{l|}{ Número de sílaba } & \multicolumn{2}{c|}{ Acento } & \multicolumn{2}{c|}{$\begin{array}{c}\text { Frontera } \\
\text { sintagmática }\end{array}$} \\
\cline { 3 - 11 } \multicolumn{2}{c|}{} & M & H & M & H & M & H & M & H \\
\hline \multirow{3}{*}{ PMx1 } & O & 249 & 152 & 5 & 5 & A & A & ISV & ISV \\
\cline { 2 - 11 } & P & 271 & 150 & 4 & 4 & A & A & FSN & FSN \\
\cline { 2 - 10 } & Pr & 288 & 154 & 3 & 3 & A & A & PSN & PSN \\
\hline \multirow{3}{*}{ PMx2 } & O & 231 & 138 & 8 & 7 & A & A & ISP & FSV \\
\cline { 2 - 10 } & P & 218 & 142 & 8 & 7 & A & A & ISP & FSV \\
\cline { 2 - 10 } & Pr & 208 & 135 & 7 & 7 & A & A & FSV & FSV \\
\hline
\end{tabular}

Tabla 1. Picos máximos en la modalidad declarativa en el hombre y en la mujer ${ }^{3}$

3 M: mujer; H: hombre; O: Oxítono; P: Paroxítono; Pr: Proparoxítono; A: átona; ISV: inicio del sintagma verbal; FSN: final del sintagma nominal; PSN: penúltima del sintagma nominal; FSV: final del sintagma verbal; ISP: inicio del sintagma preposicional. 
De acuerdo con los datos precedentes, extraemos tres informaciones de interés: primera, el salto de F0 hacia el PMx1 se produce siempre desde la pretónica hasta la postónica; segunda, los dos picos máximos se sincronizan siempre con una vocal átona, esto es, con la postónica en el caso del PMx1, y con esta misma sílaba o la pos-postónica en el caso del PMx2; en este punto, la mujer y el hombre se diferencian, puesto que la primera sitúa el pico en la pos-postónica, salvo en el caso de los proparoxítonos que lo coloca en la postónica, sílaba esta en la que recae el PMx2 del hombre sin excepciones. Por último, en relación con las fronteras entre sintagmas, los dos picos, con independencia de la estructura acentual, delimitan fronteras fuertes (SN/ $\mathrm{SV}$ y SV/SP), puesto que mayoritariamente se sincronizan con el inicio o final de los sintagmas implicados o, excepcionalmente en el caso de las proparoxítonas del PMx1, la altura máxima se alcanza en la penúltima del SN y, por tanto, está próximo a la misma frontera sintagmática.

En la Tabla 2 se puede ver, en St, el salto inicial desde el valle a la tónica (V-T), el rango anterior desde el valle hasta el pico (V-PMx) y posterior $(\mathrm{PMx}-\mathrm{V})$, y el escalonamiento de los dos picos (!), es decir, la diferencia entre PMx1 y PMx2.

\begin{tabular}{|c|c|c|c|c|c|c|c|c|c|c|c|c|}
\cline { 2 - 16 } \multicolumn{1}{c|}{} & \multicolumn{2}{c|}{ V-T } & \multicolumn{2}{c|}{ V-PMx1 } & \multicolumn{2}{c|}{ PMx1-V } & \multicolumn{2}{c|}{ V-PMx2 } & \multicolumn{2}{c|}{ PMx2-F } & \multicolumn{2}{c|}{ ! PMx1-2 } \\
\cline { 2 - 15 } \multicolumn{1}{c|}{} & M & H & M & H & M & H & M & H & M & H & M & H \\
\hline O & 2,4 & 3,9 & 2,7 & 4,5 & 2,2 & 4,6 & 1,4 & 4,6 & 5,2 & 10 & $-1,3$ & $-1,8$ \\
\hline P & 1,8 & 1,6 & 3,4 & 3,6 & 4,9 & 2,8 & 1,8 & 2,8 & 6,9 & 7,7 & $-3,7$ & $-0,9$ \\
\hline Pr & 2,7 & 2,9 & 6 & 5,7 & 7,1 & 4,9 & 1 & 5,5 & 2,2 & 7,1 & $-5,6$ & $-2,3$ \\
\hline
\end{tabular}

Tabla 2. Diferencias en St del rango tonal en las declarativas

De los datos de la tabla precedente se concluye lo siguiente:

$1^{\circ}$ ) El salto inicial desde el valle (situado en una sílaba átona) hasta la tónica (que, como dijimos, no se sincroniza con el pico puesto que este se retrasa hasta la postónica) supera siempre el umbral perceptivo de $1,5 \mathrm{St}$, con valores similares en hombre y mujer, salvo en los oxítonos, donde el primero supera a la mujer en 1,5 St.

$2^{\circ}$ ) Los rangos anteriores y posteriores de los picos rebasan, asimismo, dicho umbral, excepto en dos casos de la mujer (V-PMx2 de oxítonos y proparoxítonos).

$3^{\circ}$ ) Por último, el escalonamiento descendente de los dos picos supera el umbral perceptivo, excepto en los oxítonos de la mujer $(1,3 \mathrm{St})$ y en los paroxítonos del hombre $(0,9 \mathrm{St})$. 
Teniendo en cuenta los resultados precedentes, hemos etiquetado los tres acentos tonales de las frases analizadas y el tono de frontera final según la propuesta de Dorta (ed.) (2013: 72), realizada a partir de la notación $S p$ ToBI (Spanish Tones and Break Indices). Los resultados se muestran en las Tablas 3 (nivel superficial o fonético) y 4 (nivel profundo o fonológico).

\begin{tabular}{|c|c|c|c|c|c|c|c|c|c|c|c|c|}
\hline & \multicolumn{3}{|c|}{ Acento 1 (SN) } & \multicolumn{3}{|c|}{ Acento 2 (SV) } & \multicolumn{3}{|c|}{$\begin{array}{c}\text { Acento } 3 \\
\text { (Sprep) }\end{array}$} & \multicolumn{3}{|c|}{$\begin{array}{c}\text { Tono de } \\
\text { frontera final }\end{array}$} \\
\hline & $\mathrm{O}$ & $\mathrm{P}$ & $\operatorname{Pr}$ & $\mathrm{O}$ & $\mathrm{P}$ & $\operatorname{Pr}$ & $\mathrm{O}$ & $\mathrm{P}$ & $\operatorname{Pr}$ & $\mathrm{O}$ & $\mathrm{P}$ & $\operatorname{Pr}$ \\
\hline 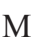 & $\mathrm{L}+>\mathrm{H}^{*}$ & $\mathrm{~L}+>\mathrm{H}^{*}$ & $\mathrm{~L}+$ & $\mathrm{L}^{*}$ & $\mathrm{~L}^{*+}$ !H & $\mathrm{L}^{*}$ & $\mathrm{~L}^{*}$ & $\mathrm{~L}^{*}$ & $L^{*}$ & $\mathrm{~L} \%$ & $\mathrm{~L} \%$ & $\mathrm{~L} \%$ \\
\hline 1 & $\mathrm{~L}+>\mathrm{H}^{*}$ & $\mathrm{~L}+>\mathrm{H}^{*}$ & $\mathrm{~L}+>\mathrm{H}^{*}$ & $+! \mathrm{H}$ & $L^{*+H}$ & $\mathrm{~L}^{*+! \mathrm{H}}$ & $\mathrm{L}^{*}$ & $\mathrm{~L}^{*}$ & $\mathrm{~L}^{*}$ & L\% & $\mathrm{L} \%$ & $\mathrm{~L} \%$ \\
\hline
\end{tabular}

Tabla 3. Interpretación fonética de los acentos tonales y tonos de frontera de las oraciones declarativas

\begin{tabular}{|c|c|c|c|c|}
\cline { 2 - 5 } \multicolumn{1}{c|}{} & Acento 1 (SN) & Acento 2 (SV) & $\begin{array}{c}\text { Acento 3 } \\
\text { (Sprep) }\end{array}$ & $\begin{array}{c}\text { Tono de } \\
\text { frontera final }\end{array}$ \\
\hline $\mathrm{M}$ & $/ \mathrm{L}+\mathrm{H}^{* /}$ & $\mathrm{L}^{*} / \mathrm{L}^{*}+\mathrm{H}$ & $\mathrm{L}^{*}$ & $\mathrm{~L} \%$ \\
\hline $\mathrm{H}$ & $/ \mathrm{L}+\mathrm{H}^{* /}$ & $\mathrm{L}^{*}+\mathrm{H}$ & $\mathrm{L}^{*}$ & $\mathrm{~L} \%$ \\
\hline
\end{tabular}

Tabla 4. Interpretación fonológica de los acentos tonales y tonos de frontera de las oraciones declarativas

Como puede verse, desde el punto de vista fonológico, los dos informantes coinciden en presentar un acento tonal inicial $/ \mathrm{L}+\mathrm{H}^{*} /$ debido a que, siguiendo la propuesta de Dorta, este acento se describe como un "acento bitonal ascendente con tónica significativamente alta respecto del valle anterior. El ascenso desde el valle al pico es también significativo" (2013: 73), circunstancias estas que se dan en nuestro estudio (Tabla 2). Debido a que el pico se retrasa hasta la postónica en todos los casos, la única variante de este acento es $\left[\mathrm{L}+>\mathrm{H}^{*}\right]$ (Tabla 3 ). En el SV, se da el acento monotonal $/ \mathrm{L}^{*} /$, realizado como $\left[\mathrm{L}^{*}\right]$, y el bitonal $/ \mathrm{L}^{*}+\mathrm{H} /$, realizado como $\left[\mathrm{L}^{*}+\mathrm{H}\right] \mathrm{o}\left[\mathrm{L}^{*+}+\mathrm{H}\right]$, diferenciándose la mujer y el hombre en que en este último solo se registra el acento bitonal. En ambos acentos, el valle se da en la tónica y el descenso desde el pico precedente supera el umbral (Tabla 2); la diferencia entre el acento monotonal y el bitonal es que, en el primero el ascenso desde el valle, situado en la tónica, al segundo pico no es significativo, mientras que en el bitonal sí lo es. Cuando el escalonamiento entre los picos supera el umbral perceptivo, este escalonamiento se indica en la realización fonética pero no en 
la fonológica puesto que consideramos que $\left[\mathrm{L}^{*}+\mathrm{H}\right]$ y $\left[\mathrm{L}^{*}+\right.$ ! H] son variantes $\mathrm{de} / \mathrm{L}^{*}+\mathrm{H} /$. Finalmente, las declarativas, como es usual en el español general, presentan un tono nuclear descendente $/ \mathrm{L}^{*} /$ con la realización $\left[\mathrm{L}^{*}\right]$ ya que la acentuada se coloca en un tono bajo debido al progresivo descenso de F0 por lo que, asimismo, terminan con un tono de juntura bajo $/ \mathrm{L} \% /$.

\subsubsection{Modalidad interrogativa}

Las interrogativas (Figura 2), a diferencia de las declarativas, tienen contornos distintos en el hombre y en la mujer: en esta última, las oxítonas y proparoxítonas son bicumbres, mientras que las paroxítonas son tricumbres. En el hombre, en cambio, las paroxítonas y proparoxítonas son bicumbres y las oxítonas monocumbres.

El inicio medio de las interrogativas de la mujer se sitúa en $217 \mathrm{~Hz}$ y el del hombre en $128 \mathrm{~Hz}$; en ambos casos, la F0 experimenta un ascenso progresivo que sobrepasa el $\mathrm{TM}^{4}$ de cada informante. Este ascenso, en los tres acentos, se inicia en la mujer a partir de la tónica que, por tanto, a diferencia de las declarativas, permanece baja, y culmina en el PMx1 que se sincroniza con una sílaba átona diferente en los tres acentos: en la última del SV de los oxítonos (FSV), en la inicial del SV de los paroxítonos (ISV) y en la última del $\mathrm{SN}$ de los proparoxítonos (FSN). En el hombre, en cambio, la F0 comienza a ascender a partir de la primera sílaba átona de cada sintagma y culmina siempre en la postónica, sea del SN o del SV (Tabla 5), tal como sucedía en las declarativas.

A partir del PMx1, se produce un descenso de F0: en los oxítonos de la voz femenina culmina en la pretónica del SPrep (sílaba 10), desde donde vuelve a ascender hasta la tónica nuclear (T), donde se da el PMx2; a partir de este sufre de nuevo un descenso que queda truncado al no poder progresar debido a que no hay sílabas átonas posteriores en este acento. En el hombre, la configuración tonal, como se dijo, es monocumbre y, por tanto, no presenta el final circunflejo descrito en la mujer, por lo que el descenso desde el PMx1, que se da en el prenúcleo, continúa hasta el final.

En las paroxítonas y proparoxítonas de ambos sexos, el descenso desde el PMx 1 termina en la tónica del SV (sílaba 6), por lo que esta tiene un tono bajo, como sucedía en las declarativas. Desde dicha sílaba, la F0 vuelve a subir, culminando en un segundo pico (PMx2), que en las paroxítonas de la mujer se sincroniza con la átona inicial del SPrep, mientras que en las proparoxítonas se da en la tónica nuclear. En estos dos mismos acentos, el

$4 \quad$ El TM es 228 y $143 \mathrm{~Hz}$ en mujer y hombre, respectivamente. 
PMx2 del hombre coincide con la átona final del SV, desde donde la F0 desciende hasta el final, como sucede en las proparoxítonas de las mujeres. En las paroxítonas de la voz femenina, se da un tercer pico (PMx3) que coincide con la tónica del núcleo.

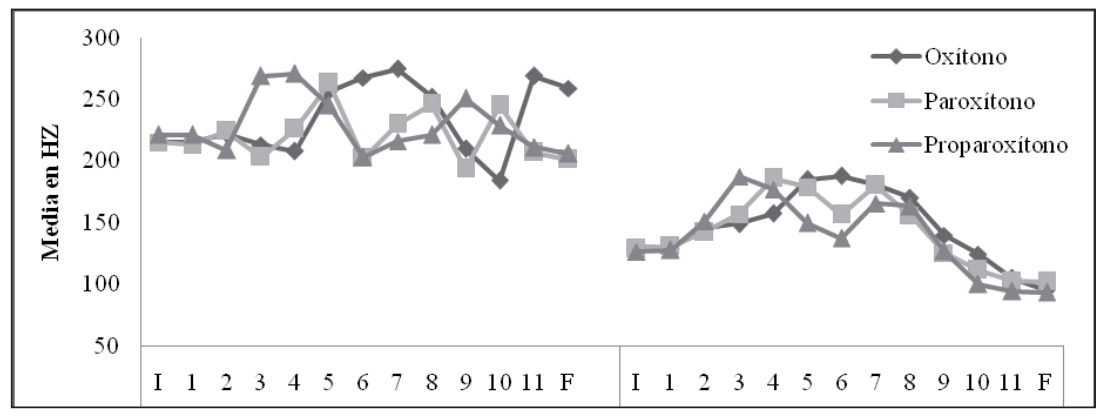

Figura 2. Modalidad interrogativa en función de la tipología acentual

\begin{tabular}{|c|c|c|c|c|c|c|c|c|c|}
\hline & & \multicolumn{2}{|c|}{$\begin{array}{l}\text { Valor } \\
\text { en HZ }\end{array}$} & \multicolumn{2}{|c|}{$\begin{array}{l}\text { Número } \\
\text { de sílaba }\end{array}$} & \multicolumn{2}{|c|}{ Acento } & \multicolumn{2}{|c|}{$\begin{array}{c}\text { Frontera } \\
\text { sintagmática }\end{array}$} \\
\hline & & M & $\mathrm{H}$ & $\mathrm{M}$ & $\mathrm{H}$ & $\mathrm{M}$ & $\mathrm{H}$ & $\mathrm{M}$ & $\mathrm{H}$ \\
\hline \multirow[t]{3}{*}{ PMx1 } & Oxítono & 275 & 188 & 7 & $5=6$ & $\mathrm{~A}$ & $\mathrm{~A}$ & FSV & $\begin{array}{l}\text { ISV/ } \\
\text { ISV }\end{array}$ \\
\hline & Paroxítono & 263 & 186 & 5 & 4 & $\mathrm{~A}$ & $\mathrm{~A}$ & ISV & FSN \\
\hline & Proparoxítono & 271 & 187 & $3=4$ & 3 & $\mathrm{~A}$ & $\mathrm{~A}$ & FSN & ISN \\
\hline \multirow[t]{3}{*}{ PMx2 } & Oxítono & 269 & -- & 11 & -- & $\mathrm{T}$ & $\mathrm{A}$ & FSP & -- \\
\hline & Paroxítono & 246 & 180 & 8 & 7 & $\mathrm{~A}$ & $\mathrm{~A}$ & ISP & FSV \\
\hline & Proparoxítono & 251 & 165 & 9 & 7 & $\mathrm{~T}$ & $\mathrm{~A}$ & FSP & FSV \\
\hline PMx3 & Paroxítono & 245 & -- & 10 & -- & $\mathrm{T}$ & -- & FSP & -- \\
\hline
\end{tabular}

Tabla 5. Picos máximos en la modalidad interrogativa en el hombre y en la mujer

En definitiva, los datos precedentes señalan algunas diferencias entre la voz femenina y masculina, pero lo más relevante es que las configuraciones tonales de las interrogativas, sean monocumbres, bicumbres o tricumbres, terminan con descenso, como es característico en algunas variedades del español, como la canaria o la cubana. Ahora bien, frente a la mujer que presenta un pico tonal nuclear que coincide con la tónica desde donde desciende la F0 hasta el final, coincidiendo así con la configuración circunfleja típica de las variedades citadas, el PMx2 en el hombre se adelanta, por lo que la tónica coincide con un tono bajo. Hay que destacar, además, que el final circunflejo, como se ha dicho más arriba, queda truncado en 
los oxítonos de la voz femenina, pero no así en la voz masculina, puesto que el único pico que se da en este caso recae en el prenúcleo, por lo que el descenso de F0 puede progresar desde el pico hasta el final. Por otra parte, en relación con las fronteras sintagmáticas, lo más relevante es que, como en la modalidad declarativa, el PMx1 delimita la frontera fuerte SN/SV.

En la Tabla 6 se puede ver, en St, el salto inicial desde el valle a la tónica (V-T) cuando el primero recae en una átona ${ }^{5}$, los rangos anteriores y posteriores de los picos, el salto del pico a la tónica nuclear -cuando aquel recae en una átona-, del último pico al final y, por último, el escalonamiento de los picos inicial y final.

\begin{tabular}{|c|c|c|c|c|c|c|c|c|c|c|c|c|c|c|c|c|c|c|}
\hline & \multicolumn{2}{|c|}{ I-T } & \multicolumn{2}{|c|}{ V-PMx1 } & \multicolumn{2}{|c|}{ PMx1-V } & \multicolumn{2}{|c|}{ V-PMx2 } & \multicolumn{2}{|c|}{ PMx2-V } & \multicolumn{2}{|c|}{ V-PMx3 } & \multicolumn{2}{|c|}{ PMx-T } & \multicolumn{2}{|c|}{$\begin{array}{l}\text { PMx 1, } \\
2 \text { o 3-F }\end{array}$} & \multicolumn{2}{|c|}{$\begin{array}{l}\text { !PMx1- } \\
\text { PMxF }\end{array}$} \\
\hline & $\mathrm{M}$ & 11 & IV & $\mathrm{H}$ & 1 & II & IV & 11 & & & & & & 11 & $\mathrm{M}$ & $\mathrm{H}$ & $\mathrm{M}$ & $\mathrm{H}$ \\
\hline $\mathrm{O}$ & -- & 3,6 & & 6 & & -- & 6 , & -- & - & -- & - & -- & -- & 12 & 0,6 & 11,8 &, 3 & -- \\
\hline $\mathrm{P}$ & -- & 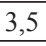 & & 5 & & 3 & & 2,4 & & 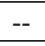 & & & -- & 8,3 & 43 & 9.83 & ,2 & $-0,2$ \\
\hline $\operatorname{Pr}$ & \begin{tabular}{l|l}
-- \\
\end{tabular} & 2,8 & 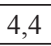 & 7 & 5 & | & 3,7 & 3,2 & -- & -- & -- & 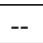 & \begin{tabular}{|l|}
-- \\
\end{tabular} & 4,4 & 3.42 & 8.33 & 0,1 & $-2,3$ \\
\hline
\end{tabular}

Tabla 6. Diferencias en St del rango tonal en las interrogativas

Como puede verse, todos los rangos tonales que hemos considerado, salvo raras excepciones, arrojan resultados diferenciales por encima del umbral perceptivo. Teniendo en cuenta los datos de la Tabla 6 y las descripciones precedentes, hemos etiquetado fonética (Tabla 7) y fonológicamente (Tabla 8) los acentos tonales de las interrogativas siguiendo la misma propuesta que en hemos adoptado en las declarativas (Dorta (ed.) 2013: 73).

\begin{tabular}{|c|c|c|c|c|c|c|c|c|c|c|c|c|}
\hline & \multicolumn{3}{|c|}{ Acento $1(\mathrm{SN})$} & \multicolumn{3}{|c|}{ Acento 2 (SV) } & \multicolumn{3}{|c|}{ Acento 3 (Sprep) } & \multicolumn{3}{|c|}{$\begin{array}{c}\text { Tono de } \\
\text { frontera final }\end{array}$} \\
\hline & $\mathrm{O}$ & $P$ & $\operatorname{Pr}$ & $O$ & $P$ & $\operatorname{Pr}$ & $\mathrm{O}$ & $P$ & $\operatorname{Pr}$ & $\bar{O}$ & $P$ & $\operatorname{Pr}$ \\
\hline $\mathrm{M}$ & $\mathrm{L}^{*+\mathrm{H}}$ & $\mathrm{L}^{*}+\mathrm{H}$ & $\mathrm{L}^{*}+\mathrm{H}$ & $\mathrm{H}^{*}$ & $*+\mathrm{H}$ & $\mathrm{L}^{*}+\mathrm{H}$ & $\mathrm{L}+\mathrm{H}^{*}$ & $\mathrm{~L}+\mathrm{H}^{*}$ & $\mathrm{~L}+\mathrm{H}^{*}$ & HL \% & $\mathrm{L} \%$ & $\mathrm{~L} \%$ \\
\hline $\mathrm{H}$ & $\mathrm{L}+>\mathrm{H}^{*}$ & $\mathrm{~L}+>\mathrm{H}^{*}$ & $\mathrm{~L}+>\mathrm{H}^{*}$ & $\mathrm{H}^{*}$ & $2^{*}+\mathrm{H}$ & $\mathrm{L}^{*+\mathrm{H}}$ & $\mathrm{L}^{*}$ & $\mathrm{~L}^{*}$ & $\mathrm{~L}^{*}$ & L $\%$ & $\mathrm{~L} \%$ & L\% \\
\hline
\end{tabular}

Tabla 7. Interpretación fonética de los acentos tonales y tonos de frontera de las oraciones interrogativas

5 Recuérdese que el valle se da en la tónica en el caso de la mujer; en el hombre se da en la átona inicial. 


\begin{tabular}{|c|c|c|c|c|}
\cline { 2 - 5 } \multicolumn{1}{c|}{} & Acento 1 (SN) & Acento 2 (SV) & $\begin{array}{c}\text { Acento 3 } \\
\text { (Sprep) }\end{array}$ & $\begin{array}{c}\text { Tono de } \\
\text { frontera final }\end{array}$ \\
\hline $\mathrm{M}$ & $/ \mathrm{L}^{*}+\mathrm{H} /$ & $\mathrm{H}^{*} / \mathrm{L}^{*}+\mathrm{H}$ & $\mathrm{H}^{*}$ & $\mathrm{~L} \%$ \\
\hline $\mathrm{H}$ & $/ \mathrm{L}+\mathrm{H}^{*} /$ & $\mathrm{H}^{*} / \mathrm{L}^{*}+\mathrm{H}$ & $\mathrm{L}^{*}$ & $\mathrm{~L} \%$ \\
\hline
\end{tabular}

Tabla 8. Interpretación fonológica de los acentos tonales y tonos de frontera de las oraciones interrogativas

Teniendo en cuenta la interpretación fonológica de la Tabla 8, es obvio que los acentos de frontera distancian a los dos informantes: en el acento inicial, la mujer utiliza un acento bitonal ascendente con tónica baja y pico posterior $/ \mathrm{L}^{*}+\mathrm{H} /$; en el hombre, en cambio, si bien tiene igualmente un acento bitonal ascendente, la tónica es significativamente alta respecto del valle anterior $/ \mathrm{L}+\mathrm{H}^{*} /$. Tal diferencia fonológica implica una clara diferencia fonética: debido al desplazamiento del pico, la variante es $\left[\mathrm{L}+>\mathrm{H}^{*}\right]$ en el hombre, mientras que en la mujer es $\left[\mathrm{L}^{*}+\mathrm{H}\right]$. En lo que respecta al núcleo, los dos informantes se apartan radicalmente, puesto que la mujer presenta un tono alto $/ \mathrm{H}^{*} /$ con la variante $\left[\mathrm{L}+\mathrm{H}^{*}\right]$ debido a que la F0 permanece alta en la tónica y desde el valle precedente se da una diferencia por encima del umbral perceptivo (Tabla 6); en cambio, el acento nuclear en el hombre es $/ \mathrm{L}^{*} /$ con la variante $\left[\mathrm{L}^{*}\right]$ debido a que, como en las declarativas, la tónica tiene un tono bajo.

En la Tabla 9 se puede ver el resultado conjunto de la interpretación fonológica de las declarativas e interrogativas del hombre y de la mujer.

\begin{tabular}{|c|c|c|c|c|c|c|c|c|}
\hline & \multicolumn{2}{|c|}{ Acento $1(\mathrm{SN})$} & \multicolumn{2}{|c|}{ Acento $2(\mathrm{SV})$} & \multicolumn{2}{|c|}{$\begin{array}{c}\text { Acento } 3 \\
\text { (Sprep) }\end{array}$} & \multicolumn{2}{|c|}{$\begin{array}{c}\text { Tono de } \\
\text { frontera final }\end{array}$} \\
\hline & $\mathrm{M}$ & $\mathrm{H}$ & $\mathrm{M}$ & $\mathrm{H}$ & $\mathrm{M}$ & $\mathrm{H}$ & $\mathrm{M}$ & $\mathrm{H}$ \\
\hline $\mathrm{D}$ & $/ \mathrm{L}+\mathrm{H}^{*} /$ & $/ \mathrm{L}+\mathrm{H}^{*} /$ & $\mathrm{L} * / \mathrm{L} *+\mathrm{H}$ & $\mathrm{L}^{*}+\mathrm{H}$ & $\mathrm{L}^{*}$ & $\mathrm{~L}^{*}$ & $\mathrm{~L} \%$ & $\mathrm{~L} \%$ \\
\hline I & $/ \mathrm{L} *+\mathrm{H} /$ & $/ \mathrm{L}+\mathrm{H}^{*} /$ & $\mathrm{H}^{*} / \mathrm{L}^{*}+\mathrm{H}$ & $/ \mathrm{H}^{*} / \mathrm{L} *+\mathrm{H}$ & $\mathrm{H}^{*}$ & $\mathrm{~L}^{*}$ & $\mathrm{~L} \%$ & $\mathrm{~L} \%$ \\
\hline
\end{tabular}

Tabla 9. Comparación de los acentos tonales y tonos de frontera de las oraciones declarativas e interrogativas

Según esta interpretación, las declarativas e interrogativas de la mujer se diferencian claramente en el acento inicial y en el nuclear. Por el contrario, en el caso del hombre, la interpretación fonológica es idéntica en ambas modalidades, por lo que habría que concluir, a falta de nuevos análisis, que la diferencia entre ellas se establece por el nivel tonal en que se sitúan, más alto en las interrogativas que en las declarativas, salvo excepcionalmente en el final de los proparoxítonos, como se puede ver en las Figuras 3-5. 


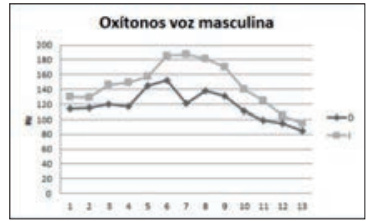

Figura 3

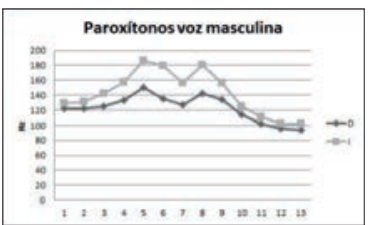

Figura 4

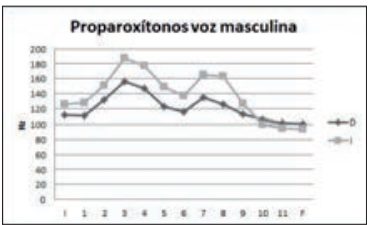

Figura 5

Figuras 3-5. Modalidad declarativa e interrogativa en voz masculina de Medellín

En efecto, en la tabla 10 se puede ver la diferencia tonal entre las dos modalidades a favor de la modalidad interrogativa, excepto en las sílabas 10,11 y $\mathrm{F}$ de los proparoxítonos (que marcamos con el signo negativo), en donde los valores sitúan a las interrogativas por debajo de las declarativas.

\begin{tabular}{|c|c|c|c|c|c|c|c|c|c|c|c|c|c|}
\cline { 2 - 15 } \multicolumn{1}{c|}{} & $\mathrm{I}$ & 1 & 2 & 3 & 4 & 5 & 6 & 7 & 8 & 9 & 10 & 11 & $\mathrm{~F}$ \\
\hline $\mathrm{O}$ & 2,3 & 2 & 3,4 & 4,2 & 1,4 & 3,4 & 7,6 & 4,7 & 4,5 & 4 & 4 & 1,7 & 1,9 \\
\hline $\mathrm{P}$ & 1 & 1,2 & 2,2 & 2,9 & 3,7 & 4,9 & 3,6 & 4,1 & 2,6 & 1,6 & 1,5 & 1 & 1,4 \\
\hline $\mathrm{Pr}$ & 2 & 2,5 & 2,3 & 3 & 3,2 & 3,4 & 2,9 & 3,4 & 4,4 & 2 & -1 & $-1,4$ & $-1,2$ \\
\hline
\end{tabular}

Tabla 10. Diferencias en St entre la modalidad declarativa e interrogativa, a favor de esta última, en voz masculina

Como se puede ver, las diferencias a favor de la modalidad interrogativa superan, salvo raras excepciones, el umbral perceptivo de 1,5 St, por lo que cabe suponer que tales diferencias son suficientes para discriminar auditivamente ambas modalidades, aun cuando los acentos tonales presenten idéntico patrón fonológico.

\subsection{RELACIÓN DEL INICIO Y FINAL DE LAS CONFIGURACIONES TONALES CON EL TONO MEDIO Y PENDIENTES TONALES INICIO-FINAL}

\subsubsection{Inicio y tono medio}

En las oraciones declarativas, los inicios de la mujer se sitúan por encima del tono medio y los del hombre por debajo; sin embargo, las diferencias entre el inicio y el tono medio en ambos sexos son irrelevantes desde el punto de vista perceptivo. En las oraciones interrogativas coinciden hombre y mujer en situar los valores por debajo del tono medio; sin embargo, mientras que en la mujer las diferencias no son relevantes en los tres acentos, en el hombre sí lo son, sobre todo en las proparoxítonas (Figura 6). 


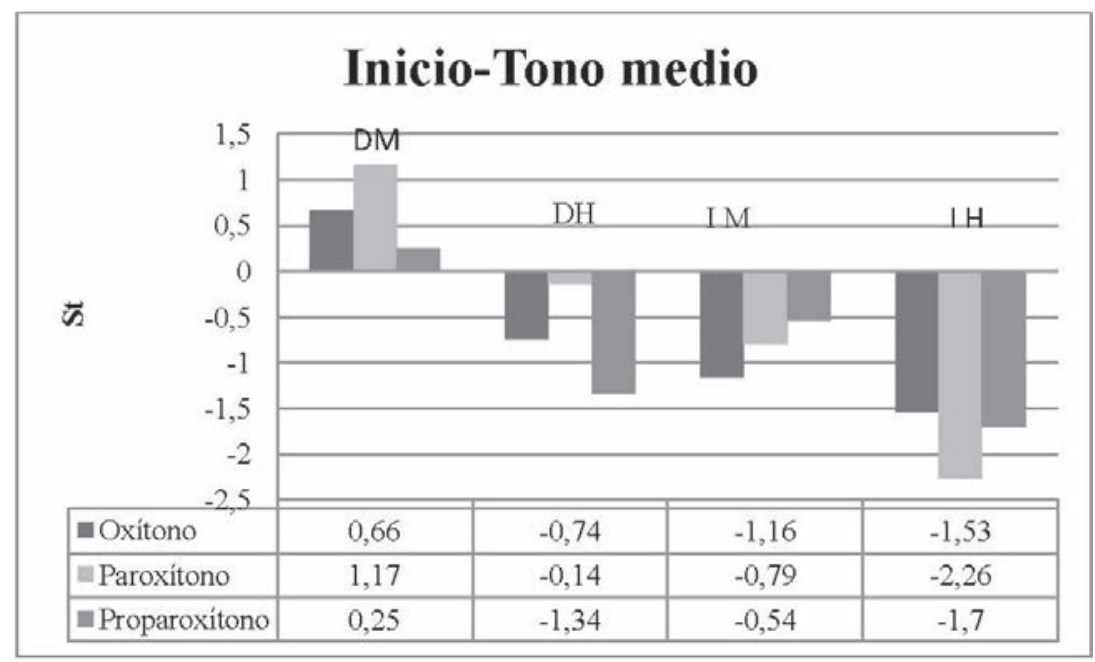

Figura 6. Inicio y tono medio en las modalidades declarativa e interrogativa ${ }^{6}$

\subsubsection{Final y tono medio}

Debido a la peculiaridad de los finales descendentes de la modalidad interrogativa, el final de la F0 se sitúa significativamente, desde el punto de vista perceptivo, por debajo del TM en las dos modalidades analizadas, con la excepción de las oraciones interrogativas, en donde los finales oxítonos de la mujer se mantienen por encima del TM debido al truncamiento final que se da en los mismos y que ya comentamos más arriba (Figura 7).

6 D e I: declarativas e interrogativas; $\mathrm{M}$ y H: mujer y hombre. 


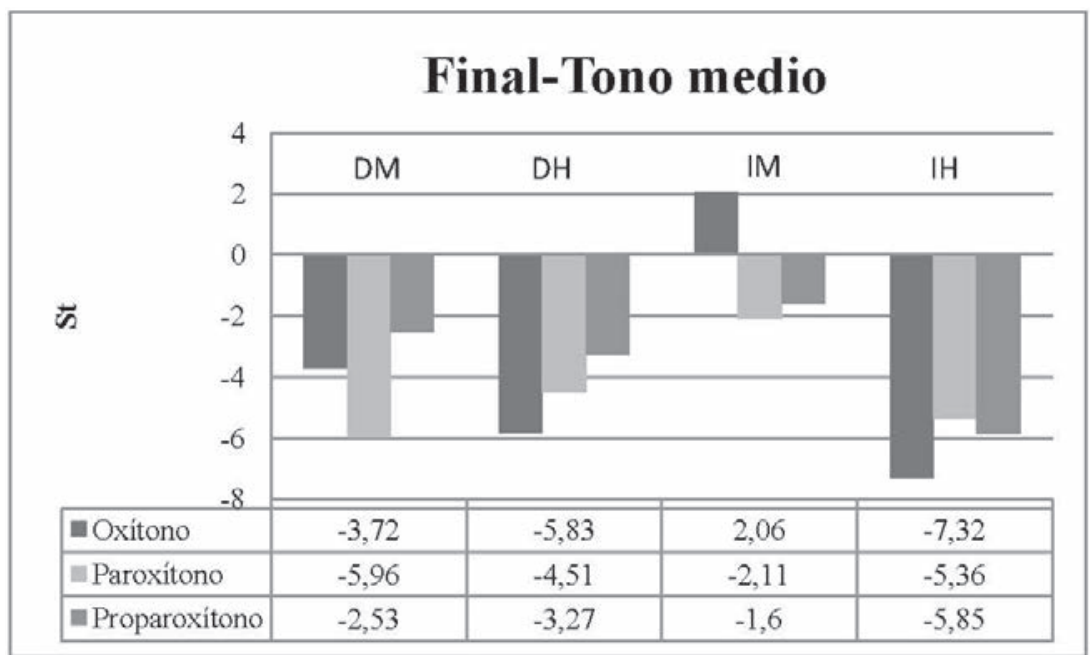

Figura 7. Final y tono medio en las modalidades declarativa e interrogativa

\subsubsection{Pendiente Inicio-Final}

La pendiente I-F en las dos modalidades y en los dos sexos (Figura 5) es descendente, excepto en las oxítonas interrogativas de la mujer que es ascendente por las razones ya explicadas.

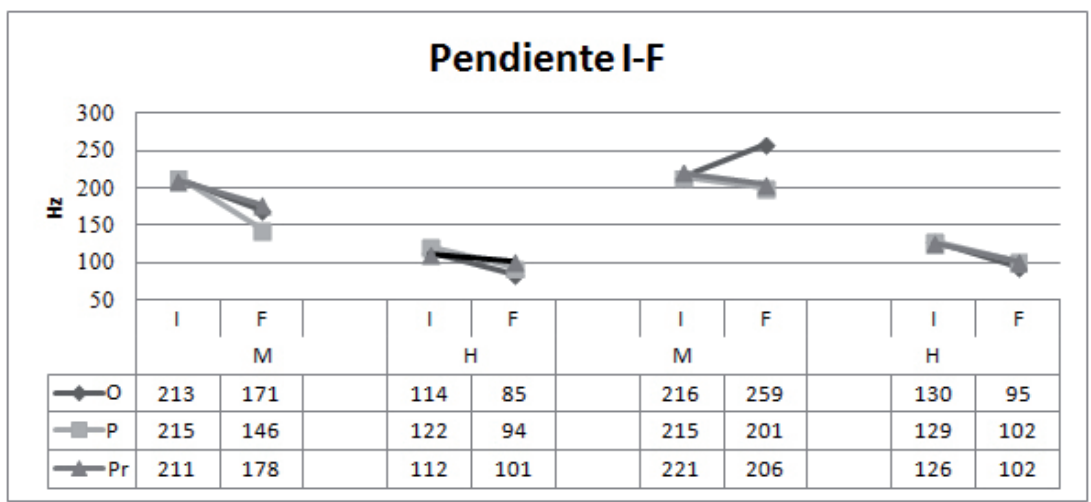

Figura 8. Pendiente I-F en las modalidades declarativa e interrogativa

Las diferencias, en St, entre el inicio y el final en cada informante, así como las diferencias entre los dos sexos (M-H) pueden verse en la tabla 5. 


\begin{tabular}{|c|c|c|c|c|c|c|}
\cline { 2 - 7 } \multicolumn{1}{c|}{} & \multicolumn{3}{c|}{$\mathrm{D}$} & \multicolumn{2}{c|}{$\mathrm{I}$} \\
\cline { 2 - 7 } \multicolumn{1}{c|}{} & $\mathrm{M}$ & $\mathrm{H}$ & $\mathrm{M}-\mathrm{H}$ & $\mathrm{M}$ & $\mathrm{H}$ & $\mathrm{M}-\mathrm{H}$ \\
\hline $\mathrm{O}$ & $-3,80$ & $-5,08$ & 1,28 & 3,14 & $-5,43$ & 2,29 \\
\hline $\mathrm{P}$ & $-6,70$ & $-4,51$ & 2,19 & $-1,17$ & $-4,07$ & 2,9 \\
\hline $\mathrm{Pr}$ & $-2,94$ & $-1,79$ & 1,15 & $-1,22$ & $-3,66$ & 2,44 \\
\hline
\end{tabular}

Tabla 11. Valores de pendiente, en St, en H y M y diferencia entre los dos sexos

Como puede comprobarse, en las declarativas, los oxítonos del hombre presentan una pendiente descendente más pronunciada que los de la mujer; en cambio, en los otros dos acentos sucede lo contrario. Con todo, la diferencia de pendiente entre los dos sexos solo supera el umbral en los paroxítonos, a favor de la mujer. En las interrogativas, en cambio, los valores de pendiente son siempre más acusados en el hombre, por lo que las diferencias con respecto a la mujer son siempre significativas. Además, como se puede ver en la Figura 8, en el hombre no se registra una pendiente ascendente en los oxítonos, como sucede en la mujer.

\section{CONCLUSIONES Y DISCUSIÓN DE LOS RESULTADOS}

El objetivo principal de este estudio era comparar la entonación de la voz femenina y masculina de dos hablantes colombianos pertenecientes a la zona urbana de Medellín. El estudio nos ha permitido observar lo siguiente:

1. Respecto a la tipología acentual y las fronteras sintagmáticas en función de la modalidad oracional.

a) La voz femenina y masculina presenta en las oraciones declarativas configuraciones tonales bicumbres, como es común en castellano (Quilis 1993: 428) y, específicamente, en el bogotano (Sosa 1999: 187-192). Dicha estructura se caracteriza por dos picos máximos. Sin embargo, la mujer y el hombre se diferencian, puesto que la primera sitúa el PMx2 en la pos-postónica, salvo en el caso de los proparoxítonos que lo coloca en la postónica, sílaba esta en la que se sitúa el PMx2 del hombre sin excepciones, como lo ha sugerido Sosa (1999) para el español. Las oraciones son descendentes como en términos generales se sugiere para la mayoría de lenguas (Gårding 1985). 
b) En las oraciones interrogativas, las configuraciones tonales continúan siendo bicumbres, excepto en la paroxítona de la mujer que es tricumbre y en la oxítona del hombre que es monocumbre. A pesar de lo anterior, todas las oraciones finalizan con descenso, como es característico en algunas variedades del español, como la canaria (Dorta ed. 2013 [zona urbana]; Hernández, Díaz y Jorge 2014 [zona rural]) o la cubana (Dorta ed. 2013; García Riverón 1996; Sosa 1999). Por tanto, la voz del hombre y de la mujer se alejan de la entonación ascendente sugerida para Bogotá por Sosa (1999) y por Quilis (1993).

2. Teniendo en cuenta el umbral psicoacústico de 1,5 St para realizar el etiquetaje de las curvas (Dorta ed. 2013), destacamos el comportamiento tonal en los dos acentos de frontera y en el tono de juntura final:

a) En el acento inicial, los dos participantes coinciden en presentar en la modalidad declarativa un acento bitonal $/ \mathrm{L}+\mathrm{H}^{*} /$. En la modalidad interrogativa, en cambio, la mujer tiene un acento bitonal ascendente con tónica baja y pico posterior $/ \mathrm{L}^{*}+\mathrm{H} /$, mientras que el hombre presenta igualmente un acento bitonal ascendente, pero con tónica significativamente alta respecto del valle anterior $/ \mathrm{L}+\mathrm{H} * /$.

b) En el SV de las declarativas se da el acento monotonal /L*/ y el bitonal $/ \mathrm{L}^{*}+\mathrm{H} /$, diferenciándose la mujer y el hombre en que en este último solo se da el acento bitonal. En las interrogativas, el hombre y la mujer coinciden en un acento bitonal con tónica baja y pico posterior $/ \mathrm{L}^{*}+\mathrm{H} /$ en paroxítonas y proparoxítonas, pero, en cambio, en las oxítonas presentan un acento monotonal alto $/ \mathrm{H} * /$.

c) En las declarativas, el acento nuclear es bajo $/ \mathrm{L} * /$, debido al progresivo descenso de $\mathrm{F}$, lo que determina, asimismo, un tono de juntura bajo /L\%/. En las interrogativas, los dos participantes se apartan radicalmente, puesto que en la mujer se da un tono alto $/ \mathrm{H}^{*} /$; en cambio, el acento nuclear en el hombre es $/ \mathrm{L}^{*} /$. En ambos, el tono de juntura es $/ \mathrm{L} \% /$.

d) En definitiva, en la mujer, las declarativas e interrogativas se diferencian claramente en el acento inicial y en el nuclear. Por el contrario, en el hombre, la interpretación fonológica es idéntica en ambas modalidades. Sin embargo, a pesar de las coincidencias, es fácil identificar perceptivamente una oración declarativa de una interrogativa, por lo cual, a falta de otros análisis, atribuimos las diferencias al nivel tonal en que se sitúan ambas modalidades, más alto en las interrogativas que en las declarativas. 
e) Con respecto a las fronteras sintagmáticas, lo más relevante es que se observa una relación entre el límite prosódico y el límite sintagmático fuerte en ambas modalidades: el PMx1 delimita la frontera fuerte SN/SV y el PMX2 la frontera fuerte SV/SP. La mujer presenta un tercer pico en las proparoxítonas que se ubica en el SP. La relación comentada, sin duda, se ve favorecida por el tipo de corpus analizado; habría que ver si se corrobora en corpus más espontáneos.

3. Respecto al nivel inicial y final del contorno entonativo en relación con el tono medio encontramos lo siguiente:

a) Mientras que en el hombre el inicio en ambos tipos de modalidades se sitúa por debajo del TM, en la mujer se coloca por encima en las declarativas y por debajo en las interrogativas. Además, solamente hay diferencias significativas en las interrogativas del hombre.

b) A diferencia del hombre, la voz femenina sitúa los finales de los oxítonos por encima del TM.

4. La pendiente I-F, en términos generales, es descendente en la voz femenina y masculina, es decir, los finales se sitúan siempre por debajo de los inicios, independientemente de la modalidad, excepto en las interrogativas oxítonas de la voz femenina, en las que se sitúa por encima.

\section{CONCLUSIÓN GENERAL}

Sin olvidar que este es un estudio preliminar de la voz femenina y masculina, podemos sugerir que ambas voces presentan varias coincidencias, pero también rasgos que las tipifican. Entre estos últimos tenemos que la frecuencia de la voz femenina es significativamente más alta que la masculina, como era obvio esperar. La mujer sitúa el PMx2 de las declarativas en la pospostónica, mientras que el hombre lo hace en la postónica. En las oraciones interrogativas, la mujer presenta un contorno tricumbre en la paroxítona y el hombre un contorno monocumbre en las oxítonas. El último PMx de la mujer coincide con la última tónica, mientras que en el hombre recae en vocal átona. En la voz femenina, las configuraciones del contorno tonal de ambas modalidades se diferencian en el prenúcleo y en el núcleo, mientras que en el hombre la diferencia se centra en el nivel tonal, más alto en las interrogativas que en las declarativas. En la comparación de los inicios y 
el TM solamente hay diferencias significativas en las interrogativas del hombre, cuyo inicio se sitúa por debajo del TM. En la pendiente I-F, el inicio se sitúa por encima del final solamente en el caso de las interrogativas oxítonas de la mujer.

\section{REFERENCIAS BIBLIOGRÁFICAS}

Dorta, Josefa. (ed.). 2013. Estudio comparativo preliminar de la entonación de Canarias, Cuba y Venezuela. Madrid/Tenerife: La Página ediciones S/L.

García Riverón, Raquel. 1996. Aspectos de la entonación hispánica. I Metodología. II Análisis acústico de muestras del español de Cuba. III Las funciones de la entonación en el español de Cuba. Cáceres: Servicio de Publicaciones de la Universidad de Extremadura.

G̊̊rding, Eva. 1985. Comparing intonation. Working Papers 27: 75-96.

Gelfand, Stanley. 1981. Hearing: an introduction to psychological and physiological acoustics. New York: M. Dekker.

Hernández, Beatriz, Chaxiraxi Díaz Cabrera y Carolina Jorge Trujillo. 2014. Declarativas e interrogativas en zonas rurales de Canarias. En Yolanda Congosto Martín, María Luisa Montero Curiel y Antonio Salvador Plans (eds.). Fonética Experimental, Educación Superior e Investigación, T. III, pp. 245-265. Madrid: Editorial Arco Libros.

Ham, Lorena. 2003. Entonación: índice de identidad dialectal. Bogotá: Uniandes. (Documentos CESO).

KLATT, Denis. 1973. Discrimination of fundamental frequency contours in synthetic speech: implication for models of speech perception. Journal of acoustical Society of America 53: 8-16.

López, María, Carmen Muñiz, Liliana Díaz, Norberto Corral, David Brezmes y Mercedes Alvarellos. 2007. Análisis y representación de la entonación. Replanteamiento metodológico en el marco del proyecto AMPER. En Josefa Dorta (ed.). La prosodia en el ámbito lingüístico románico, pp. 17-34. Tenerife: La Página Ediciones, S. L. Universidad.

Nooteвоom, Sieb. 1997. The prosody of speech: Melody and rhythm. En Hardcastle, William y John Laver (eds.). The Handbook of Phonetic Sciences, pp. 640-673. Oxford: Blackwell.

Osorio, Matilde y Diana MuÑoz. 2011. La entonación del enunciado interrogativo en el español de la ciudad de Medellín. Lingüistica y Literatura 60: 209-275.

Pamies Bertrán, Antonio, Ana María Fernández Planas, Eugenio Martínez Celdrán, Alicia Ortega Escandell y Mari Cruz Amorós Céspedes. 2002. Umbrales tonales en español peninsular. En Jesús Díaz García (ed.). Actas del II Congreso de Fonética Experimental. Sevilla, pp. 272-278.

Pollack, Irwin. 1952. The information of elementary auditory displays. Journal of the Acoustical Society of America 24: 745-749.

Quilis, Antonio. 1993. Tratado de fonología y fonética españolas. Madrid: Gredos.

Rietveld, A., C. M. y Carlos Gussenhoven. 1985. On the relation between pitch excursion size and prominence. Journal of Phonetics 13: 299-308.

Romano, Antonio y Michel Contini. 2001. Un progetto di Atlante geoprosódico multimediale delle varieta linguistiche romanze. En Magno Caldognetto, Pelachaud y Piero Cosi, Multimodalità e Multimedialità nella Comunicazione, Atti delle XI Giornate di Studio del 
Gruppo di Fonetica Sperimentale, dell'Ass pp. 121-126. Italiana di Acustica (Padova, 9 Nov. $-1^{\circ}$ Dic. 2000), Padova: Unipress,

Sosa, Juan Manuel. 1999. La entonación del español. Su estructura fónica,variabilidad y dialectología. Madrid: Cátedra.

Toledo, Guillermo. 2000. Taxonomía tonal del español. Language Design 3: 1-20.

\section{ANEXO}

Se presenta a continuación el corpus analizado, indicando entre paréntesis su identificación. Cada una de las frases, como se dijo ya, fue repetida tres veces.

1. La guitarra se toca con paciencia

(kwt)

2. El bandolín se toca con paciencia (pwt)

3. La máquina se toca con paciencia (twk)

4. La guitarra se toca con emoción (kwk)

5. El bandolín se toca con emoción (pwk)

6. La máquina se toca con emoción (twp)

7. La guitarra se toca con pánico (kwp)

8. El bandolín se toca con pánico (pwp)

9. La máquina se toca con pánico 